\title{
A Geometric Approach to Low-Rank Matrix Completion
}

\author{
Wei Dai*, Ely Kerman**, Olgica Milenkovic* \\ *Department of Electrical and Computer Engineering, ${ }^{* *}$ Department of Mathematics \\ University of Illinois at Urbana-Champaign \\ Email: \{weidai07,ekerman,milenkov\}@illinois.edu
}

\begin{abstract}
The low-rank matrix completion problem can be succinctly stated as follows: given a subset of the entries of a matrix, find a low-rank matrix consistent with the observations. While several low-complexity algorithms for matrix completion have been proposed so far, it remains an open problem to devise search procedures with provable performance guarantees for a broad class of matrix models. The standard approach to the problem, which involves the minimization of an objective function defined using the Frobenius metric, has inherent difficulties: the objective function is not continuous and the solution set is not closed. To address this problem, we consider an optimization procedure that searches for a column (or row) space that is geometrically consistent with the partial observations. The geometric objective function is continuous everywhere and the solution set is the closure of the solution set of the Frobenius metric. We also preclude the existence of local minimizers, and hence establish strong performance guarantees, for special completion scenarios, which do not require matrix incoherence or large matrix size.
\end{abstract}

\section{INTRODUCTION}

In many practical applications of data acquisition, the signals of interest have a sparse representation in some basis. That is, they can be well approximated using only a few basis elements. This allows for efficient sampling and reconstruction of signals [1], [2], [3], [4], [5], [6]. More precisely, the number of linear measurements required to capture a sparse signal can be much smaller than the number of inherent dimensions of the signal, and various polynomial time algorithms are known for accurately reconstructing the sparse signal based on these linear measurements. Due to the significant reduction in sampling resources and modest requirements for computational resources, sparse signal processing has been studied intensively [1], [2], [3], [4], [5], [6].

There are two categories of sparse signals which frequently arise in applications. In the first category, the sparse signal can be modeled a vector with only a small fraction of non-zero entries. Compressive sensing is the framework of sampling and recovering such signals. In the second category, the signals are represented by matrices whose ranks are much smaller than either of their dimensions. In the second setting, one of the fundamental problems of sparse signal processing is the lowrank matrix completion problem - to determine when and how one can recover a low-rank matrix based on only a subset of its entries [5], [6], [7].

Scores of methods and algorithms have been proposed for low-rank matrix completion. Many of them are based on sim- ilarities between compressive sensing reconstruction and lowrank matrix completion. In general, both reconstruction tasks are ill-posed and computationally intractable. Nevertheless, exact recovery in an efficient manner is possible for both signal categories provided that the signal is sufficiently sparse or sufficiently densely sampled. Casting the sparse signal recovery problem as an optimization problem, $\ell_{1}$-minimization has been proposed for compressive sensing signal reconstruction [1], [2], [3]. Following the same idea, methods based on nuclear norm minimization have been developed for low-rank matrix completion [5], [6], [8], [9]. In terms of greedy algorithms, many of the approaches for low-rank completion can be viewed as generalizations of their counterparts for compressive sensing reconstruction. In particular, the ADMiRA algorithm [10] is a counterpart of the subspace pursuit (SP) [11] and CoSaMP [12] algorithms, while the singular value projection (SVP) method [13] extends the iterative hard thresholding (IHT) [14] approach. There are also other approaches that utilize some special structural properties of the low-rank matrices. Examples include the power factorization algorithm [15], the OptSpace algorithm [16], and the subspace evolution and transfer algorithm [17].

Nevertheless, there is a fundamental problem in low-rank matrix completion which has not been successfully addressed yet: how to search for a low-rank matrix consistent with partial observations. The fundamental difference between compressive sensing and low-rank matrix completion lies in the knowledge of the "sparse basis". In compressive sensing, the basis under which the signal is sparse is known a priori. In principle, the support set of the nonzero entries can be found by exhaustive search. However, in low-rank matrix completion, the corresponding "sparse basis" is not known. Note that the set of all possible bases forms a continuous space. In such a space, "exhaustive" search is impossible. Moreover, we shall show, in Example 1 of Section IIII that a direct gradientdescent search does not work either.

The understanding of the search for consistent matrices is incomplete. There are two special cases where specially designed algorithms can guarantee a consistent low-rank solution. The first case is when the low-rank matrix is fully sampled. The consistent low-rank solution is simply the observation matrix itself. The corresponding "sparse basis" (singular vectors) can be easily obtained by a singular value decomposition. The other case is when the rank equals to one. Given an arbitrary sampling pattern, one simply looks at the ratios 
between the revealed entries in the same column and uses these ratios to construct a column vector that represents the column space. This method is guaranteed to find a consistent solution for rank-one matrices. However, it remains an open problem how to extend this method for general ranks. Hence, such an approach is not universal. On the other hand, none of existing general algorithms provides performance guarantee even for the rank-one case. The performance guarantee of nuclear norm minimization is built on incoherence conditions, which only holds with high probability when the low-rank matrix is drawn randomly from certain ensembles and when the size of the matrix is sufficiently large. Our understanding of low-rank matrix completion is far from complete.

Our approach to address these issues is summarized as follows.

1) We provide a framework for searching for a low-rank matrix that is consistent with the partial observations. There is no requirement that such a matrix is unique: if there is a unique low-rank solution, we should be able to find this unique matrix; otherwise, it suffices to find just one solution that agrees with the revealed entries. In our approach, we assume that the rank of the underlying low-rank matrix is known a priori. Finding a consistent low-rank matrix is equivalent to finding a consistent column/row space. This is different from the OptSpace algorithm in [16], where the search is performed on both column and row spaces simultaneously.

2) We propose a geometric performance metric to measure the consistency between the estimated column space and the partial observations. In the literature, the standard approach is to minimize an objective function that is defined via the Frobenius norm. As we shall illustrate with explicit examples, this objective function may have singularities, and therefore the corresponding solution set may not be closed. Hence, we introduce a new formulation where consistency is now defined in geometric terms. This allows us to address the difficulties related to the Frobenius metric. In particular, we show that our geometric objective function is always continuous. The set of the corresponding consistent solutions is the closure of the set corresponding to the Frobenius norm. This new metric allows for provably strong performance guarantees, described below.

3) We provide strong performance guarantees for special completion scenarios: rank-one matrices with arbitrary sampling patterns, and fully sampled matrices 1 of arbitrary rank. For these two scenarios, a gradient descent search starting from a random point will converge to a global minimum with probability one. More importantly, if the partial observations admit a unique consistent solution, this search procedure finds this unique solution with probability one. The performance guarantees are different from those previously established in literature. Roughly speaking, previous performance guaran-

\footnotetext{
${ }^{1}$ For full sampled matrices, even though using a simple singular value decomposition produces a consistent column space, it is not clear that a randomlly initialized search would converge to a consistent column space. In what follows, we prove that this is the case.
}

tees require large matrix sizes and only hold with high probability. Ours hold with probability one regardless of matrix size. It is also worth noting that we do not require incoherence conditions, which are essential for the performance guarantees of nuclear norm minimization. Unfortunately, we are presently unable to obtain performance guarantees for more general cases.

The paper is organized as follows. In Section [I] we introduce the low-rank matrix completion problem, and some background material regarding Grassmann manifolds and their geometry. In Section III we show that formulating the lowrank matrix completion problem as an optimization problem using the Frobenius norm may yield singularities which can obstruct standard minimization algorithms. We then propose a new geometric formulation of the problem as a remedy to this difficulty. This new formulation allows for strong performance guarantees that are presented in Section IV. Section $\nabla$ summarizes the main contributions of the work. Proofs of the main results are presented in the Appendices.

\section{LOW-RAnK MATRIX COMPLETION AND PRELIMINARIES}

Let $\boldsymbol{X} \in \mathbb{R}^{m \times n}$ be an unknown matrix with rank $r \leq$ $\min (m, n)$, and let $\Omega \subset[m] \times[n]$ be the set of indices of the observed entries, where $[K]=\{1,2, \cdots, K\}$. Define the projection operator $\mathcal{P}_{\Omega}$ by

$$
\begin{aligned}
\mathcal{P}_{\Omega}: & \mathbb{R}^{m \times n} \rightarrow \mathbb{R}^{m \times n} \\
& \mathcal{P}_{\Omega}(\boldsymbol{X}) \mapsto \boldsymbol{X}_{\Omega}, \text { where }\left(\boldsymbol{X}_{\Omega}\right)_{i, j}=\left\{\begin{array}{ll}
\boldsymbol{X}_{i, j} & \text { if }(i, j) \in \Omega \\
0 & \text { if }(i, j) \notin \Omega
\end{array} .\right.
\end{aligned}
$$

The consistent matrix completion problem is to find one rank- $r$ matrix $\boldsymbol{X}^{\prime}$ that is consistent with the observations $\boldsymbol{X}_{\Omega}$, i.e.,

$$
\begin{aligned}
& (P 0): \text { find a } \boldsymbol{X}^{\prime} \text { such that } \\
& \qquad \operatorname{rank}\left(\boldsymbol{X}^{\prime}\right)=r \text { and } \mathcal{P}_{\Omega}\left(\boldsymbol{X}^{\prime}\right)=\mathcal{P}_{\Omega}(\boldsymbol{X})=\boldsymbol{X}_{\Omega} .
\end{aligned}
$$

By definition, this problem is well defined since $\boldsymbol{X}_{\Omega}$ is obtained from some rank- $r$ matrix $\boldsymbol{X}$ which is therefore a solution. As in other works, [10], [15], [16], we assume that the rank $r$ is given. In practice, one may try to sequentially guess a rank bound until a satisfactory solution has been found.

We also introduce the (standard) projection operator $\mathcal{P}$,

$$
\begin{aligned}
\mathcal{P}: \mathbb{R}^{m} \times \mathbb{R}^{m \times k} & \rightarrow \mathbb{R}^{m} \\
\mathcal{P}(\boldsymbol{x}, \boldsymbol{U}) & \mapsto \boldsymbol{y}=\boldsymbol{U} \boldsymbol{U}^{\dagger} \boldsymbol{x},
\end{aligned}
$$

where $1 \leq k \leq m$, and where the superscript $\dagger$ denotes the pseudoinverse of a matrix. Let $\operatorname{span}(\boldsymbol{U})$ denote the subspace spanned by the columns of the matrix $\boldsymbol{U}$, i.e.,

$$
\operatorname{span}(\boldsymbol{U})=\left\{\boldsymbol{v} \in \mathbb{R}^{m}: \boldsymbol{v}=\boldsymbol{U} \boldsymbol{w} \text { for some } \boldsymbol{w} \in \mathbb{R}^{m}\right\} .
$$

One can describe $\mathcal{P}(\boldsymbol{x}, \boldsymbol{U})$, in geometric terms, as the projection of the vector $\boldsymbol{x}$ onto span $(\boldsymbol{U})$. It should be observed that $\boldsymbol{U}^{\dagger} \boldsymbol{x}$ is the global minimizer of the quadratic optimization problem $\min _{\boldsymbol{w} \in \mathbb{R}^{k}}\|\boldsymbol{x}-\boldsymbol{U} \boldsymbol{w}\|_{2}^{2}$. 


\section{A. Search for a consistent column space}

We now show that the problem $(P 0)$ is equivalent to finding a column space consistent with the observed entries of $\boldsymbol{X}$.

Let $\mathcal{U}_{m, r}$ be the set of $m \times r$ matrices with $r$ orthonormal columns, i.e., $\mathcal{U}_{m, r}=\left\{\boldsymbol{U} \in \mathbb{R}^{m \times r}: \boldsymbol{U}^{T} \boldsymbol{U}=\boldsymbol{I}_{r}\right\}$. Define the function $f_{F}: \mathcal{U}_{m, r} \rightarrow \mathbb{R}$ by setting

$$
f_{F}(\boldsymbol{U})=\min _{\boldsymbol{W} \in \mathbb{R}^{n \times r}}\left\|\boldsymbol{X}_{\Omega}-\mathcal{P}_{\Omega}\left(\boldsymbol{U} \boldsymbol{W}^{T}\right)\right\|_{F}^{2},
$$

where $\|\cdot\|_{F}$ denotes the Frobenius norm. This function measures the consistency between the matrix $\boldsymbol{U}$ and the observations $\boldsymbol{X}_{\Omega}$. In particular, if $f_{F}(\boldsymbol{U})=0$, then there exists a matrix $\boldsymbol{W}$ such that the rank-r matrix $\boldsymbol{U} \boldsymbol{W}^{T}$ satisfies $\mathcal{P}_{\Omega}\left(\boldsymbol{U} \boldsymbol{W}^{T}\right)=\boldsymbol{X}_{\Omega}$. Hence, the consistent matrix completion problem is equivalent to

$$
(P 1) \text { : find } \boldsymbol{U} \in \mathcal{U}_{m, r} \text { such that } f_{F}(\boldsymbol{U})=0 .
$$

In fact, $f_{F}(U)$ depends only on the subspace span $(\boldsymbol{U})$ since the columns of a matrix of the form $\boldsymbol{U} \boldsymbol{W}^{T}$ all lie in span $(\boldsymbol{U})$. Hence, to solve the consistent matrix completion problem, it suffices to find a column space that is consistent with the observed entries. Note that the same conclusion holds for the row space as well. For simplicity, we restrict our attention to the column space only.

\section{B. Grassmann Manifolds}

The set of column spaces of elements in $\mathcal{U}_{m, r}$ can be identified with the Grassmann manifold $\mathcal{G}_{m, r}$, the set of $r$ dimensional subspaces in the $m$-dimensional Euclidean space $\mathbb{R}^{m}$. This is a smooth compact manifold of dimension $r(m-$ $r)$. Conversely, every element, say $\mathscr{U} \in \mathcal{G}_{m, r}$ can be presented by a generator matrix $\boldsymbol{U} \in \mathcal{U}_{m, r}$ satisfying $\operatorname{span}(\boldsymbol{U})=\mathscr{U}$. However, this presentation of $\mathscr{U}$ by a generator matrix is clearly not unique. Nevertheless, it follows from the discussion in the previous section that the function $f_{F}$ descends to a function on $\mathcal{G}_{m, r}$. Thus, problem $(P 1)$ can be viewed as an optimization problem on the compact manifold $\mathcal{G}_{m, r}$.

In this section we recall some facts concerning the geometry of Grassmann manifolds which will be useful in addressing this and similar optimization problems. For the proofs of these facts the reader is referred to [18]. We begin by recalling the construction of the standard Riemannian metric, $g_{m, r}$, on $\mathcal{G}_{m, r}$. Note that the group $\mathcal{U}_{m, m}$ of orthogonal $m \times m$ matrices acts transitively on $\mathcal{G}_{m, r}$ (by multiplication on generator matrices). More precisely, $\mathcal{G}_{m, r}$ can be described as a quotient of $\mathcal{U}_{m, m}$, i.e.,

$$
\mathcal{G}_{m, r}=\mathcal{U}_{m, m} /\left(\mathcal{U}_{m-r, m-r} \times \mathcal{U}_{r, r}\right)
$$

Now, as a compact Lie group, $\mathcal{U}_{m, m}$ has a standard (biinvariant) Riemannian metric (can be defined by using inner product in the tangent space). This descends to the quotient $\mathcal{G}_{m, r}$ as the metric $g_{m, r}$. By construction, $g_{m, r}$ is invariant under the action of $\mathcal{U}_{m, m}$.

The metric $g_{m, r}$ determines a chordal distance function and geodesic curves on $\mathcal{G}_{m, r}$ which will play an important role in what follows. To obtain the relevant formulas for these objects we require the notion of the principal angles between two subspaces [19], [20]. Consider the subspaces $\operatorname{span}(\boldsymbol{U})$ and $\operatorname{span}(\boldsymbol{V})$ of $\mathbb{R}^{m}$ for some $\boldsymbol{U} \in \mathcal{U}_{m, p}$ and $\boldsymbol{V} \in \mathcal{U}_{m, q}$. The principal angles between these two subspaces can be defined in the following constructive manner. Without loss of generality, assume that $1 \leq p \leq q \leq m$. Let $\boldsymbol{u}_{1} \in \operatorname{span}(\boldsymbol{U})$ and $\boldsymbol{v}_{1} \in$ $\operatorname{span}(\boldsymbol{V})$ be unit-length vectors such that $\left|\boldsymbol{u}_{1}^{T} \boldsymbol{v}_{1}\right|$ is maximal. Inductively, let $\boldsymbol{u}_{k} \in \operatorname{span}(\boldsymbol{U})$ and $\boldsymbol{v}_{k} \in \operatorname{span}(\boldsymbol{V})$ be unit vectors such that $\boldsymbol{u}_{k}^{T} \boldsymbol{u}_{j}=0$ and $\boldsymbol{v}_{k}^{T} \boldsymbol{v}_{j}=0$ for all $1 \leq j<k$ and $\left|\boldsymbol{u}_{k}^{T} \boldsymbol{v}_{k}\right|$ is maximal. The principal angles are then defined as

$$
\alpha_{k}=\arccos \boldsymbol{u}_{k}^{T} \boldsymbol{v}_{k}
$$

for $k=1,2, \cdots, p$.

Alternatively, the principal angles can be computed via singular value decomposition. Consider the singular value decomposition $\boldsymbol{U} \boldsymbol{U}^{T} \boldsymbol{V} \boldsymbol{V}^{T}=\overline{\boldsymbol{U}} \boldsymbol{\Lambda} \overline{\boldsymbol{V}}^{T}$, where $\overline{\boldsymbol{U}} \in \mathcal{U}_{m, p}$ and $\overline{\boldsymbol{V}} \in \mathcal{U}_{m, p}$ contain the first $p$ left and right singular vectors, respectively, and $\boldsymbol{\Lambda} \in \mathbb{R}^{p \times p}$ is a diagonal matrix comprised of singular values $\lambda_{1} \geq \cdots \geq \lambda_{p}$. Then the $k^{t h}$ columns of $\overline{\boldsymbol{U}}$ and $\overline{\boldsymbol{V}}$ correspond to the vectors $\boldsymbol{u}_{k}$ and $\boldsymbol{v}_{k}$ used in the constructive definition, respectively. The $k^{t h}$ singular value $\lambda_{k}$ defines the $k^{t h}$ principal angle $\alpha_{k}$ via

$$
\cos \alpha_{k}=\lambda_{k} \text {. }
$$

Chordal distance on $\mathcal{G}_{m, r}$. For $\boldsymbol{U}_{1}$ and $\boldsymbol{U}_{2}$ in $\mathcal{U}_{m, r}$, the chordal distance between the two subspaces $\operatorname{span}\left(\boldsymbol{U}_{1}\right)$ and $\operatorname{span}\left(\boldsymbol{U}_{2}\right)$ in $\mathcal{G}_{m, r}$ is given, in terms of the $p$ principal angles between them, via the formula

$$
\sqrt{\sum_{k=1}^{r} \sin ^{2} \alpha_{k}}
$$

The chordal distance can also be expressed in terms of singular values as

$$
\sqrt{\sum_{k=1}^{r}\left(1-\lambda_{k}^{2}\right)}
$$

Geodesics on $\mathcal{G}_{m, r}$. We will use the gradient descent method on $\mathcal{G}_{m, r}$ to search for consistent column spaces. This will require some information concerning the geodesics of the metric $g_{m, r}$ on $\mathcal{G}_{m, r}$ which we now recall.

Roughly speaking, a geodesic in a manifold is a generalization of the notion of a straight line in the Euclidean space: given any two points in $\mathcal{G}_{m, r}$, among all curves that connect these two points, the one of the shortest length is geodesic. More precisely, fix a subspace $\mathscr{U}$ in $\mathcal{G}_{m, r}$ and a tangent vector $\mathscr{H}$ to $\mathcal{G}_{m, r}$ at $\mathscr{U}$. Let $\boldsymbol{U} \in \mathcal{U}_{m, r}$ be a generator matrix for $\mathscr{U}$. The tangent space to $\mathcal{G}_{m, r}$ at $\mathscr{U}$ can be identified with the set of horizontal tangent vectors to $\boldsymbol{U}$, i.e., the set of tangent vectors $\boldsymbol{W}$ at $\boldsymbol{U}$ which satisfy $\boldsymbol{U}^{T} \boldsymbol{W}=0$ [18]. Let $\boldsymbol{H} \in \mathbb{R}^{m \times r}$ be the horizontal tangent vector at $\boldsymbol{U}$ which corresponds to $\mathscr{H}$ and set

$$
\boldsymbol{U}(t)=\left[\boldsymbol{U} \boldsymbol{V}_{H}, \boldsymbol{U}_{H}\right]\left[\begin{array}{c}
\cos \left(\boldsymbol{S}_{H} t\right) \\
\sin \left(\boldsymbol{S}_{H} t\right)
\end{array}\right] \boldsymbol{V}_{H}^{T},
$$

where $\boldsymbol{U}_{H} \boldsymbol{S}_{H} \boldsymbol{V}_{H}^{T}$ is the compact singular value decomposition of $\boldsymbol{H}$. Then span $(\boldsymbol{U}(t))$ is the unique geodesic of $g_{m, r}$ which starts at $\mathscr{U}$ with "initial velocity" $\mathscr{H}$. 
We now use this general solution for the geodesic flow of $g_{m, r}$ to establish the following technical result concerning geodesics between a given pair of subspaces.

Lemma 1: Fix two elements $\boldsymbol{U}_{1}$ and $\boldsymbol{U}_{2}$ of $\mathcal{U}_{m, r}$. Let $\boldsymbol{V}_{1} \boldsymbol{\Lambda} \boldsymbol{V}_{2}^{T}$ be the singular value decomposition of the matrix $\boldsymbol{U}_{1}^{T} \boldsymbol{U}_{2}$, and denote the $i^{\text {th }}$ singular value by $\lambda_{i}=\cos \alpha_{i}$. Set $\overline{\boldsymbol{U}}_{1}=\boldsymbol{U}_{1} \boldsymbol{V}_{1}$ and $\overline{\boldsymbol{U}}_{2}=\boldsymbol{U}_{2} \boldsymbol{V}_{2}$ and note that $\overline{\boldsymbol{U}}_{1}^{T} \overline{\boldsymbol{U}}_{2}=\boldsymbol{\Lambda}$.

1) Consider the path

$$
\boldsymbol{U}(t)=\left[\overline{\boldsymbol{U}}_{1}, \boldsymbol{G}\right]\left[\begin{array}{c}
\operatorname{diag}\left(\left[\cdots, \cos \alpha_{i} t, \cdots\right]\right) \\
\operatorname{diag}\left(\left[\cdots, \sin \alpha_{i} t, \cdots\right]\right)
\end{array}\right] \boldsymbol{V}_{1}^{T},
$$

where the columns of $\boldsymbol{G}=\left[\cdots, \boldsymbol{g}_{i}, \cdots\right] \in \mathbb{R}^{m \times r}$ are defined as follows

$$
\boldsymbol{g}_{i}= \begin{cases}\frac{\overline{\boldsymbol{U}}_{2,: i}-\lambda_{i} \overline{\boldsymbol{U}}_{1,: i}}{\left\|\overline{\boldsymbol{U}}_{2,: i}-\lambda_{i} \overline{\boldsymbol{U}}_{1, i}\right\|} & \text { if } \lambda_{i} \neq 1, \\ \mathbf{0} & \text { if } \lambda_{i}=1 .\end{cases}
$$

Here, the subscript $: i$ denotes the $i^{\text {th }}$ column of the corresponding matrix. Then the path $\operatorname{span}(\boldsymbol{U}(t))$ is a geodesic of $g_{m, r}$ such that $\operatorname{span}(\boldsymbol{U}(0))=\operatorname{span}\left(\boldsymbol{U}_{1}\right)$ and $\operatorname{span}(\boldsymbol{U}(1))=\operatorname{span}\left(\boldsymbol{U}_{2}\right)$.

2) Let $\overline{\boldsymbol{x}} \in \operatorname{span}\left(\boldsymbol{U}_{2}\right)$ be a unit-norm vector. It's clear that there exists a unique $\overline{\boldsymbol{w}} \in \mathcal{U}_{r, 1}$ such that $\overline{\boldsymbol{x}}=\overline{\boldsymbol{U}}_{2} \overline{\boldsymbol{w}}$. Suppose that $\overline{\boldsymbol{x}} \notin \operatorname{span}\left(\overline{\boldsymbol{U}}_{1}\right)$. Let $k$ the number of the singular values of $\overline{\boldsymbol{U}}_{1}^{T} \overline{\boldsymbol{U}}_{2}$ that equal to one. Then $k<r$ and there exists an index $j \in[r]$ such that $k<j \leq r$ and $\bar{w}_{j} \neq 0$.

Proof: Clearly, $\boldsymbol{U}(0)=\boldsymbol{U}_{1}$. Since $\overline{\boldsymbol{U}}_{1}^{T} \overline{\boldsymbol{U}}_{2}=\boldsymbol{\Lambda}$, we have

$$
\begin{aligned}
& \left\|\overline{\boldsymbol{U}}_{2,: i}-\lambda_{i} \overline{\boldsymbol{U}}_{1,: i}\right\|^{2} \\
& =1-2 \lambda_{i}\left\langle\overline{\boldsymbol{U}}_{2,: i}, \overline{\boldsymbol{U}}_{1,: i}\right\rangle+\lambda_{i}^{2} \\
& =1-\lambda_{i}^{2} .
\end{aligned}
$$

Thus, we have

$$
\begin{aligned}
\boldsymbol{U}(1) & =\left[\cdots, \overline{\boldsymbol{U}}_{1, i} \cos \alpha_{i}+\boldsymbol{g}_{i} \sin \alpha_{i}, \cdots\right] \boldsymbol{V}_{1}^{T} \\
& =\left[\cdots, \overline{\boldsymbol{U}}_{1, i} \lambda_{i}+\boldsymbol{g}_{i} \sqrt{1-\lambda_{i}^{2}}, \cdots\right] \boldsymbol{V}_{1}^{T} \\
& =\left[\cdots, \overline{\boldsymbol{U}}_{1, i} \lambda_{i}+\boldsymbol{g}_{i}\left\|\overline{\boldsymbol{U}}_{2,: i}-\lambda_{i} \overline{\boldsymbol{U}}_{1,: i}\right\|, \cdots\right] \boldsymbol{V}_{1}^{T} \\
& =\left(\overline{\boldsymbol{U}}_{1} \boldsymbol{\Lambda}+\left(\overline{\boldsymbol{U}}_{2}-\overline{\boldsymbol{U}}_{1} \boldsymbol{\Lambda}\right)\right) \boldsymbol{V}_{1}^{T} \\
& =\boldsymbol{U}_{2} \boldsymbol{V}_{2} \boldsymbol{V}_{1}^{T} .
\end{aligned}
$$

Hence, $\operatorname{span}(\boldsymbol{U}(1))=\operatorname{span}\left(\boldsymbol{U}_{2}\right)$. To prove the first part of the lemma it just remains to show that $\operatorname{span}(\boldsymbol{U}(t))$ is geodesic. Setting $\boldsymbol{H}=\dot{\boldsymbol{U}}(0)$ we have

$$
\boldsymbol{H}=\boldsymbol{G} \operatorname{diag}\left(\left[\cdots, \alpha_{i}, \cdots\right]\right) \boldsymbol{V}_{1}^{T} .
$$

We first verify that the tangent vector $\boldsymbol{H}$ is horizontal which is equivalent to showing that $\boldsymbol{U}_{1}^{T} \boldsymbol{H}=0$. According to the definition of the vectors $\boldsymbol{g}_{i}$, when $\lambda_{i} \neq 1$, one has

$$
\left\|\overline{\boldsymbol{U}}_{2,: i}-\lambda_{i} \overline{\boldsymbol{U}}_{1,: i}\right\| \neq 0
$$

and

$$
\begin{aligned}
\overline{\boldsymbol{U}}_{1}^{T} \boldsymbol{g}_{i} & =\frac{1}{\left\|\overline{\boldsymbol{U}}_{2,: i}-\lambda_{i} \overline{\boldsymbol{U}}_{1,: i}\right\|} \overline{\boldsymbol{U}}_{1}^{T}\left(\overline{\boldsymbol{U}}_{2,: i}-\lambda_{i} \overline{\boldsymbol{U}}_{1,: i}\right) \\
& =\frac{1}{\left\|\overline{\boldsymbol{U}}_{2,: i}-\lambda_{i} \overline{\boldsymbol{U}}_{1,: i}\right\|} \lambda_{i} \boldsymbol{e}_{i}-\lambda_{i} \boldsymbol{e}_{i}=\mathbf{0} .
\end{aligned}
$$

Hence,

$$
\boldsymbol{U}_{1}^{T} \boldsymbol{G}=\boldsymbol{V}_{1}^{T} \overline{\boldsymbol{U}}_{1}^{T} \boldsymbol{G}=\mathbf{0} .
$$

By (6), this implies that $\boldsymbol{U}_{1}^{T} \boldsymbol{H}=0$, as desired. Note that equation (6) can also be viewed as an expression for the compact singular value decomposition of $\boldsymbol{H}$. It then follows directly from (4) that $\operatorname{span}(\boldsymbol{U}(t))$ is indeed a geodesic.

To prove the second part of the lemma, let $\boldsymbol{u}_{1,1}, \cdots, \boldsymbol{u}_{1, r}$ and $\boldsymbol{u}_{2,1}, \cdots, \boldsymbol{u}_{2, r}$ be the column vectors of the matrix $\overline{\boldsymbol{U}}_{1}$ and $\overline{\boldsymbol{U}}_{2}$, respectively. By assumption, $\lambda_{1}=\cdots=\lambda_{k}=1$ and $1>\lambda_{k+1} \geq \cdots \geq \lambda_{r}$. Hence,

$$
\begin{aligned}
& \boldsymbol{u}_{1, j}=\boldsymbol{u}_{2, j}, \text { for all } j \leq k, \text { and } \\
& \left\langle\boldsymbol{u}_{1, j}, \boldsymbol{u}_{2, j}\right\rangle=\lambda_{j}<1, \text { for all } k<j \leq r .
\end{aligned}
$$

Suppose that $k=r$. Then

$$
\overline{\boldsymbol{x}}=\overline{\boldsymbol{U}}_{2} \overline{\boldsymbol{w}}=\overline{\boldsymbol{U}}_{2} \overline{\boldsymbol{w}} \in \operatorname{span}\left(\overline{\boldsymbol{U}}_{1}\right),
$$

which contradicts the assumption that $\overline{\boldsymbol{x}} \notin \operatorname{span}\left(\overline{\boldsymbol{U}}_{1}\right)$. Hence, we have $k<r$. Now suppose that $\bar{w}_{k+1}=\cdots=\bar{w}_{r}=0$. Then

$$
\overline{\boldsymbol{x}}=\sum_{j=1}^{k} \boldsymbol{u}_{2, j} \bar{w}_{j}=\sum_{j=1}^{k} \boldsymbol{u}_{1, j} \bar{w}_{j} \in \operatorname{span}\left(\boldsymbol{U}_{1}\right),
$$

which again contradicts the assumption that $\overline{\boldsymbol{x}} \notin \operatorname{span}\left(\boldsymbol{U}_{1}\right)$. Hence, there exists a $j$ such that $k<j \leq r$ and $\bar{w}_{j} \neq 0$. This completes the proof.

An invariant measure on $\mathcal{G}_{m, r}$. The space $\mathcal{U}_{m, m}$ admits a standard invariant measure (the Haar measure) [21]. This descends to a measure $\mu$ on $\mathcal{G}_{m, r}$ which is also invariant in the following sense: for any measurable set $\mathcal{M} \subset \mathcal{G}_{m, r}$ and any $\boldsymbol{A} \in \mathcal{U}_{m, m}$, one has $\mu(\mathcal{M})=\mu(\boldsymbol{A M})$, where $\boldsymbol{A} \mathcal{M}=$ $\left\{\operatorname{span}(\boldsymbol{A U}): \boldsymbol{U} \in \mathcal{U}_{m, r}, \operatorname{span}(\boldsymbol{U}) \in \mathcal{M}\right\}$ [21], [20]. This invariant measure defines the uniform/isotropic distribution on the Grassmann manifold. Furthermore, let $\operatorname{span}(\boldsymbol{U}) \in \mathcal{G}_{m, r}$ be fixed and $\operatorname{span}(\boldsymbol{V}) \in \mathcal{G}_{m, r}$ be drawn randomly from the isotropic distribution. The joint probability density function of the principal angles between the spans of $\boldsymbol{U}$ and $\boldsymbol{V}$ is explicitly given in [21], [22], [20], [23]. Two properties of this density function will be relevant to our later analysis: first, it is independent of the choice of $\boldsymbol{U}$; second, there is no mass point.

\section{From the Frobenius Norm to the Geometric METRIC}

In the previous section, we showed that the matrix completion problem reduces to a search for a consistent column space. In other words, one only needs to find a global minimum of the objective function $f_{F}(\boldsymbol{U})$, where

$$
f_{F}(\boldsymbol{U}) \triangleq \min _{\boldsymbol{W} \in \mathbb{R}^{r \times n}}\left\|\boldsymbol{X}_{\Omega}-\mathcal{P}_{\Omega}(\boldsymbol{U} \boldsymbol{W})\right\|_{F}^{2} .
$$

However, as we shall show in Section $\amalg I-A$, this approach has a serious drawback: the objective function (7) is not a continuous function of the variable $\boldsymbol{U}$. The discontinuity of the objective function is due to the composition of the Frobenius norm with the projection operator $\mathcal{P}_{\Omega}$. It may prevent gradient-descent-based algorithms from converging to 
a global optimum (see [17] for a detailed example). To address this issue, we propose another objective function $f_{G}(\boldsymbol{U})$ based on the geometry of the problem, detailed in Section III-B. To solve the matrix completion problem, one then needs to solve the problem

$$
(P 2) \text { : find a } \boldsymbol{U} \in \mathcal{U}_{m, r} \text { such that } f_{G}(\boldsymbol{U})=0 \text {. }
$$

where $f_{G}$ denotes the geometric metric, which is formally defined in Section III-B.

In the rest of this section, we shall show that the new objective function $f_{G}$ is a continuous function. Furthermore, we shall show that the preimage of $f_{G}(\boldsymbol{U})=0$ is the closure of the preimage of $f_{F}(\boldsymbol{U})=0$. Because of these nice properties of the geometric objective function, one can derive strong performance guarantees for gradient descent methods, as described in Section IV.

\section{A. Why the Frobenius Norm Fails}

We use an example to show that the objective function (7) based on the Frobenius norm is not continuous. Let $\boldsymbol{x}_{\Omega, i}$ be the $i^{t h}$ column of the matrix $\boldsymbol{X}_{\Omega}$. Let $\Omega_{i} \subset[m]$ be the set of indices of known entries in the $i^{t h}$ column. We use $\mathcal{P}_{\Omega, i}$ to denote the projection operator corresponding to the index set of $\Omega_{i}$. By additivity of the squared Frobenius norm, the objective function can be written as a sum of atomic functions, i.e.,

$$
\begin{aligned}
f_{F}(\boldsymbol{U}) & =\min _{\boldsymbol{W} \in \mathbb{R}^{r \times n}}\left\|\boldsymbol{X}_{\Omega}-\mathcal{P}_{\Omega}(\boldsymbol{U} \boldsymbol{W})\right\|_{F}^{2} \\
& =\sum_{i=1}^{n} \underbrace{\min _{\boldsymbol{w}_{i} \in \mathbb{R}^{r}}\left\|\boldsymbol{x}_{\Omega, i}-\mathcal{P}_{\Omega, i}\left(\boldsymbol{U} \boldsymbol{w}_{i}\right)\right\|_{F}^{2}}_{f_{F, i}(\boldsymbol{U})} .
\end{aligned}
$$

Denote the $i^{\text {th }}$ atomic function by $f_{F, i}(\boldsymbol{U})$. It can be verified that

$$
\begin{aligned}
f_{F, i}(\boldsymbol{U}) & =\min _{\boldsymbol{w} \in \mathbb{R}^{r}}\left\|\boldsymbol{x}_{\Omega, i}-\mathcal{P}_{\Omega, i}\left(\boldsymbol{U} \boldsymbol{w}_{i}\right)\right\|_{F}^{2} \\
& =\left\|\boldsymbol{x}_{\Omega, i}-\mathcal{P}\left(\boldsymbol{x}_{\Omega, i}, \boldsymbol{U}_{\Omega_{i}}\right)\right\|_{F}^{2 .}
\end{aligned}
$$

where $\boldsymbol{U}_{\Omega_{i}}=\left[\mathcal{P}_{\Omega, i}\left(\boldsymbol{u}_{1}\right), \cdots, \mathcal{P}_{\Omega, i}\left(\boldsymbol{u}_{r}\right)\right]$ and $\boldsymbol{u}_{1}, \cdots, \boldsymbol{u}_{r}$ are column vectors of the matrix $\boldsymbol{U}$. We show in the next example that an atomic function, say $f_{F, 1}(\boldsymbol{U})$, may not be continuous.

Example 1: Suppose that $\boldsymbol{x}_{\Omega, 1}=[0,1,1]^{T}$ and $\Omega_{1}=\{2,3\}$. Let $\boldsymbol{U}$ be of the form $\boldsymbol{U}=\left[\sqrt{1-2 \epsilon^{2}}, \epsilon, \epsilon\right]^{T} \in \mathcal{U}_{3,1}$ where $\epsilon \epsilon$ $[-1 / \sqrt{2}, 1 / \sqrt{2}]$. For a given $\boldsymbol{U}$, the atomic function $f_{F, 1}(\boldsymbol{U})$ is given by

$$
f_{F, 1}(\boldsymbol{U})=\min _{w \in \mathbb{R}}\left\|[0,1,1]^{T}-\mathcal{P}_{\Omega, 1}(\boldsymbol{U} w)\right\|_{F}^{2} .
$$

This is a quadratic optimization problem and can be easily solved. The optimal $w^{*}$ is given by

$$
w^{*}= \begin{cases}\frac{2}{\epsilon} & \text { if } \epsilon \neq 0, \\ 0 & \text { if } \epsilon=0\end{cases}
$$

Hence, one has

$$
f_{F, 1}(\boldsymbol{U}(\epsilon))= \begin{cases}0 & \text { if } \epsilon \in\left[-\frac{1}{\sqrt{2}}, 0\right) \cup\left(0, \frac{1}{\sqrt{2}}\right], \\ 2 & \text { if } \epsilon=0 .\end{cases}
$$
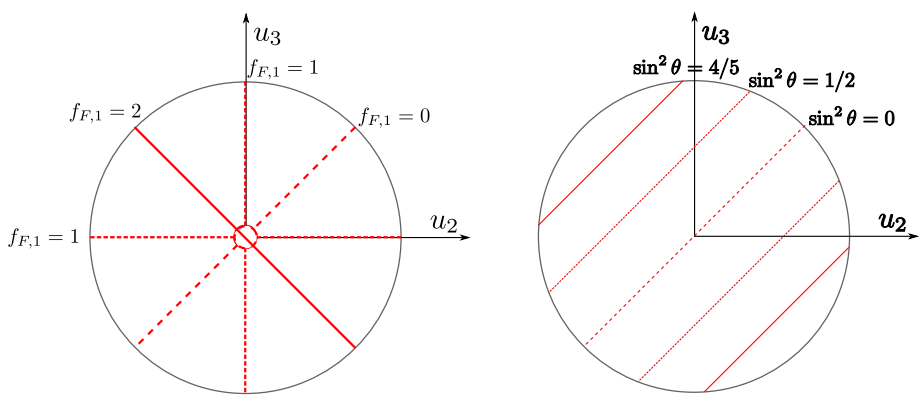

Figure 1. Contours projected to the $\left(u_{2}, u_{3}\right)$ plane. The left depicts the contours of the squared Frobenius norm. The right corresponds to the chordal distance.

which shows that $f_{F, 1}(\boldsymbol{U}(\epsilon))$ has a singular point at $\epsilon=0$.

It is straightforward to verify that the overall objective function (7) is also a discontinuous function of $\boldsymbol{U}$. As we argued in [17], this discontinuity creates so called barriers, which may prevent gradient-descent algorithms from converging to a global minimum. Hence, one seeks an optimization criteria that will allow for a continuous objective function and consequently, no search path barriers.

\section{B. A Geometric Metric}

To address the problem due to the singularities of the objective functions, we propose to replace the Frobenius norm by a geometric performance metric.

In this case, the objective function is defined as

$$
f_{G}(\boldsymbol{U})=\sum_{i=1}^{n} f_{G, i}(\boldsymbol{U})
$$

where $f_{G, i}(\boldsymbol{U})$ denotes the geometric metric corresponding to the $i^{\text {th }}$ column, defined as follows. If $\boldsymbol{x}_{\Omega, i}=\mathbf{0}$, we set $f_{G, i}(\boldsymbol{U})=0$. Henceforth, we only consider the case when $\boldsymbol{x}_{\Omega, i} \neq \mathbf{0}$. For any $\boldsymbol{x}_{\Omega, i} \neq \mathbf{0}$, let $\overline{\boldsymbol{x}}_{\Omega, i}=\boldsymbol{x}_{\Omega, i} /\left\|\boldsymbol{x}_{\Omega, i}\right\|_{F}$ be the normalized vector $\boldsymbol{x}_{\Omega, i}$. Let $\Omega_{i}^{c}=\{1,2, \cdots, m\} \backslash \Omega_{i}$ be the complement of $\Omega_{i}$. Let $\boldsymbol{e}_{k} \in \mathbb{R}^{m}$ be the $k^{t h}$ natural basis vector, i.e., the $k^{t h}$ entry of $\boldsymbol{e}_{k}$ equals to one and all other entries are zero. Define

$$
\boldsymbol{B}_{i}=\left[\overline{\boldsymbol{x}}_{\Omega, i}, \boldsymbol{e}_{k_{1}}, \cdots, \boldsymbol{e}_{k_{\ell}}\right],
$$

where $\left\{k_{1}, \cdots, k_{\ell}\right\}=\Omega_{i}^{c}$. Let $\lambda_{\max }\left(\boldsymbol{B}_{i}^{T} \boldsymbol{U}\right)$ be the largest singular value of the matrix $\boldsymbol{B}_{i}^{T} \boldsymbol{U}$. Then

$$
f_{G, i}(\boldsymbol{U})=1-\lambda_{\max }^{2}\left(\boldsymbol{B}_{i}^{T} \boldsymbol{U}\right) .
$$

This expression is closely related to the chordal distance between two subspaces, as described in Section II-B We henceforth refer to the function (10) either as the geometric metric (10), or with slight abuse of terminology, as the chordal distance.

One advantage of the chordal distance is its continuity. This follows directly from the continuity of the singular values of the underlying matrix. Recall Example 1. In Fig. 11 we illustrate the differences between $f_{F, 1}$ and $f_{G, 1}$ by projecting their contours of constant value onto the $u_{2}-u_{3}$ plane. 
More importantly, the following theorem shows that the preimage of $f_{G, i}(\boldsymbol{U})=0$ is actually the closure of the preimage of $f_{F, i}(\boldsymbol{U})=0$.

Theorem 1: Given $\boldsymbol{x}_{\Omega, i} \in \mathbb{R}^{m}$ and $\Omega_{i} \subset[\mathrm{m}]$. Let $\boldsymbol{U}_{\Omega_{i}} \in$ $\mathbb{R}^{m \times r}$ be such that $\left(\boldsymbol{U}_{\Omega_{i}}\right)_{k, \ell}=\boldsymbol{U}_{k, \ell}$ if $k \in \Omega_{i}$ and $\left(\boldsymbol{U}_{\Omega_{i}}\right)_{k, \ell}=$ 0 if $k \notin \Omega_{i}$. Define and let $\boldsymbol{b}_{i}$ and $\boldsymbol{v}_{i}$ be the corresponding left and right singular vectors, respectively 2 . Following the definition of the chordal distance, one has $f_{G, i}(\boldsymbol{U})=\sin ^{2} \theta_{i}=1-\lambda_{i}^{2}$. Let $\boldsymbol{G}_{i} \in \mathbb{R}^{m \times r}$ be a matrix such that

$$
\left(\boldsymbol{G}_{i}\right)_{k, \ell}=\frac{\partial}{\partial \boldsymbol{U}_{k, \ell}} f_{G, i}(\boldsymbol{U})=-2 \cos \theta_{i} \frac{\partial \cos \theta_{i}}{\partial \boldsymbol{U}_{k, \ell}} .
$$

$\mathcal{U}_{F, i}=\left\{\boldsymbol{U} \in \mathcal{U}_{m, r}: f_{F, i}(\boldsymbol{U})=\left\|\boldsymbol{x}_{\Omega, i}-\mathcal{P}\left(\boldsymbol{x}_{\Omega, i}, \boldsymbol{U}_{\Omega_{i}}\right)\right\|^{2}=0\right\}$ It can be verified that

and

$$
\mathcal{U}_{G, i}=\left\{\boldsymbol{U} \in \mathcal{U}_{m, r}: f_{G, i}(\boldsymbol{U})=1-\lambda_{\max }\left(\boldsymbol{B}_{i}^{T} \boldsymbol{U}\right)=0\right\} .
$$

Then $\mathcal{U}_{G, i}$ is the closure of $\mathcal{U}_{F, i}$, i.e., $\mathcal{U}_{G, i}=\overline{\mathcal{U}_{F, i}}$.

The proof is given in Appendix A Although this theorem deals with only one column of the observed matrix, the result can be easily extended to the whole matrix $\boldsymbol{X}_{\Omega}$ : let $\mathcal{U}_{F}=$ $\bigcap_{i=1}^{n} \mathcal{U}_{F, i}$ and

$$
\begin{aligned}
\mathcal{U}_{G} & =\bigcap_{i=1}^{n} \mathcal{U}_{G, i} \\
& =\left\{\boldsymbol{U} \in \mathcal{U}_{m, r}: \lambda_{\max }\left(\boldsymbol{U}^{T} \boldsymbol{B}_{i}\right)=1 \text { for all } i\right\} ;
\end{aligned}
$$

then $\mathcal{U}_{G}=\overline{\mathcal{U}_{F}}$.

Example 1 (Continued): It can be seen that

$$
\boldsymbol{B}_{1}=\left[\begin{array}{ccc}
0 & \frac{1}{\sqrt{2}} & \frac{1}{\sqrt{2}} \\
1 & 0 & 0
\end{array}\right]^{T}
$$

Hence,

$$
f_{G, 1}(\boldsymbol{U})=1-\lambda_{\max }^{2}\left(\left[\begin{array}{c}
\sqrt{2} \epsilon \\
\sqrt{1-2 \epsilon^{2}}
\end{array}\right]\right)=0 .
$$

As a result,

$$
\begin{aligned}
\mathcal{U}_{F, 1}= & \left\{\left[\sqrt{1-2 \epsilon^{2}}, \epsilon, \epsilon\right]^{T}: \epsilon^{2} \leq \frac{1}{2} \text { and } \epsilon \neq 0\right\} \\
& \bigcup\left\{\left[-\sqrt{1-2 \epsilon^{2}}, \epsilon, \epsilon\right]^{T}: \epsilon^{2} \leq \frac{1}{2} \text { and } \epsilon \neq 0\right\},
\end{aligned}
$$

and

$$
\begin{aligned}
\mathcal{U}_{G, 1}= & \left\{\left[\sqrt{1-2 \epsilon^{2}}, \epsilon, \epsilon\right]^{T}: \epsilon^{2} \leq \frac{1}{2}\right\} \\
& \bigcup\left\{\left[-\sqrt{1-2 \epsilon^{2}}, \epsilon, \epsilon\right]^{T}: \epsilon^{2} \leq \frac{1}{2}\right\} .
\end{aligned}
$$

Clearly, $\mathcal{U}_{G, 1}=\overline{\mathcal{U}_{F, 1}}$.

\section{Computations Related to the Chordal Distance}

For a given performance metric, the computational complexity of the supporting optimization procedure is an important factor for assessing its practical value. In this subsection, we show that besides its continuity, the chordal distance and the related gradient can be computed efficiently. Hence, all the algorithmic solutions using gradient descent methods can be easily modified to accommodate the geometric distortion measure.

The principal angle $\theta_{i}$ and the chordal distance $\sin ^{2} \theta_{i}$ can be computed using the singular value decomposition. Given the $i^{\text {th }}$ column of the observed matrix, one can form $\boldsymbol{B}_{i}$ easily. Let $\lambda_{i}$ be the largest singular value of the matrix $\boldsymbol{B}_{i} \boldsymbol{B}_{i}^{T} \boldsymbol{U}$,

$$
\boldsymbol{G}_{i}=-2 \lambda \boldsymbol{b}_{i} \boldsymbol{v}_{i}^{T}
$$

Note that in the matrix completion problem, one only needs to search for a column space $\operatorname{span}(\boldsymbol{U})$ consistent with the observations. Taking this fact into consideration, we have [18]

$$
\nabla_{\boldsymbol{U}} f_{G}=\sum_{i=1}^{n} \nabla_{\boldsymbol{U}} f_{G, i}=\left(\boldsymbol{I}-\boldsymbol{U} \boldsymbol{U}^{T}\right) \sum_{i=1}^{n} \boldsymbol{G}_{i} .
$$

Switching from the Frobenius norm to the chordal distance does not introduce extra computational cost. Due to the particular structure of $\boldsymbol{B}_{i}$, the matrix multiplication $\boldsymbol{B}_{i} \boldsymbol{B}_{i}^{T} \boldsymbol{U}$ can be executed in $O(m r)$ steps. The resulting matrix has dimensions $m \times r$, where typically $r \ll m$. The major computational burden is incurred by the singular value decomposition. Computing the largest singular value and the corresponding singular vectors of an $m \times r$ matrix essentially reduces to computing the largest eigenvalue of an $r \times r$ matrix and the corresponding eigenvector. Hence, the overall complexity of computing $f_{G, i}$ is $O\left(m r^{2}+r^{3}\right)=O\left(m r^{2}\right)$, where the $O\left(m r^{2}\right)$ and $O\left(r^{3}\right)$ terms come from matrix multiplication and eigenvalue computation, respectively. In comparison, to solve the least square problem in the definition of $f_{F, i}$ has a $O\left(m r^{2}\right)$ cost as well.

\section{Performance Guarantees}

Consider the matrix completion problem described in (8). The following theorem describes completion scenarios for which a global optimum can be found with probability one.

Theorem 2: Consider the following cases:

1) (rank-one matrices with arbitrary sampling): Let $\boldsymbol{X}_{\Omega}=$ $\mathcal{P}_{\Omega}(\boldsymbol{X})$ for some unknown matrix $\boldsymbol{X}$ with rank equal to one. Here, $\Omega \subset[m] \times[n]$ can be arbitrary.

2) (full sampling with arbitrary rank matrices): Let $\boldsymbol{X}_{\Omega}=$ $\boldsymbol{X}$, i.e., $\boldsymbol{\Omega}=[m] \times[n]$.

Suppose that $r=\operatorname{rank}(\boldsymbol{X})$ is given. Let $\mathcal{U}_{G} \subset \mathcal{U}_{m, r}$ be the preimage of $f_{G}(\boldsymbol{U})=0$ (also defined in (11)). Let $\boldsymbol{U}_{0}$ be randomly generated from the isotropic distribution on $\mathcal{U}_{m, r}$, and used as the initial point of the search procedure. With probability one, there exists a continuous path $\boldsymbol{U}(t), t \in[0,1]$, such that $\boldsymbol{U}(0)=\boldsymbol{U}_{0}, \boldsymbol{U}(1) \in \mathcal{U}_{G}$ and $\frac{d}{d t} f_{G} \leq 0$ for all $t \in(0,1)$, where the equality holds if and only if $\boldsymbol{U}_{0} \in \mathcal{U}_{G}$.

The proof of the theorem is outlined in Section IV-A It is worth to note that almost all starting points are good: it is certainly good if the starting point is a consistent solution;

\footnotetext{
${ }^{2}$ For convenience, we use the following convention regarding the singular vectors $\boldsymbol{b}_{i}$ and $\boldsymbol{v}_{i}$ : we let the first nonzero entry of $\boldsymbol{v}_{i}$ be positive; otherwise, we let $\boldsymbol{v}_{i}^{\prime}=-\boldsymbol{v}_{i}$ and $\boldsymbol{b}_{i}^{\prime}=-\boldsymbol{b}_{i}$, and use $\boldsymbol{v}_{i}^{\prime}$ and $\boldsymbol{b}_{i}^{\prime}$ for singular value decomposition. The simultaneous changes in signs do not affect the singular value decomposition nor the computation of the gradient.
} 
otherwise, there exists a continuous path from this starting point to a global optimum such that the objective function keeps decreasing. The performance guarantee provided in Theorem 2 is strong in the sense that it does not require either incoherence conditions or large matrix sizes.

A simple corollay of the Theorem 2 is the following result: suppose that the partial observations $\boldsymbol{X}_{\Omega}$ admit a unique consistent solution in terms of the Frobenius norm; then a gradient search procedure using the geometric norm finds this unique solution with probability one. This conclusion follows from the fact that the solution set under the Frobenius norm contains only a single point and therefore $\mathcal{U}_{G}=\overline{\mathcal{U}_{F}}=\mathcal{U}_{F}$.

For the more general case where $r>1$ and $\Omega \neq[m] \times[n]$, we can not prove the same performance guarantees. Nevertheless, in Section IV-B, we present a collection of results that may be helpful for future exploration.

\section{A. Proof of Theorem 2}

For our proof techniques, we need the following two assumptions.

Assumption I: There exists a global optimum $\boldsymbol{U}_{X} \in \mathcal{U}_{m, r}$ such that $f_{G}\left(\boldsymbol{U}_{\boldsymbol{X}}\right)=0$ and all the $r$ principal angles between $\operatorname{span}\left(\boldsymbol{U}_{X}\right)$ and $\operatorname{span}\left(\boldsymbol{U}_{0}\right)$ are less than $\pi / 2$. That is, all the singular values of $\boldsymbol{U}_{X}^{T} \boldsymbol{U}_{0}$ are strictly positive.

Assumption II: All of the $\theta_{i}$ 's (the smallest principal angle between span $\left(\boldsymbol{U}_{0}\right)$ and $\left.\operatorname{span}\left(\boldsymbol{B}_{i}\right)\right)$ are less than $\pi / 2$.

Remark 1: Suppose that the matrix $\boldsymbol{U}_{0}$ is randomly drawn from the uniform (isotropic) distribution on $\mathcal{U}_{m, r}$. Then $\boldsymbol{U}_{0}$ satisfies both assumptions with probability one. This result can be easily verified using the probability density function of the principal angles [21], [22], [20], [23].

Assuming that these two assumptions are satisfied, we have the following two theorems corresponding to the two cases in Theorem 2, respectively.

Theorem 3: (Rank-One Case) Let $\boldsymbol{X}_{\Omega}$ be the partial observation matrix generated from a rank-one matrix. Let $\boldsymbol{u}_{0} \in$ $\mathcal{U}_{m, 1}$ be an estimate of the column space that satisfies Assumptions I and II. Suppose that $\sum_{i=1}^{n} \sin ^{2} \theta_{i} \neq 0$. Then there exists a continuous path $\boldsymbol{u}(t) \in \mathcal{U}_{m, r}$ such that $\boldsymbol{u}(0)=\boldsymbol{u}_{0}$, $\boldsymbol{u}(1) \in \mathcal{U}_{G}$, and $\left.\frac{d}{d t}\right|_{t=0} \sin ^{2} \theta_{i} \leq 0$ for all $i \in[n]$, where equality holds if and only if $\theta_{i}(0)=0$.

Theorem 4: (Full-Sampling Case) Let $\boldsymbol{X} \in \mathbb{R}^{m \times n}$ be a rank-r matrix. Let $\boldsymbol{U}_{0} \in \mathcal{U}_{m, r}$ satisfy Assumptions I and II. Suppose that $\sum_{i=1}^{n} \sin ^{2} \theta_{i} \neq 0$. Then there exists a $\boldsymbol{U}(t) \in$ $\mathcal{U}_{m, r}$ such that $\boldsymbol{U}(0)=\boldsymbol{U}_{0}, \boldsymbol{U}(1) \in \mathcal{U}_{G}$ and $\left.\frac{d}{d t}\right|_{t=0} \sin ^{2} \theta_{i} \leq$ 0 for all $i \in[n]$, where equality holds if and only if $\theta_{i}(0)=0$.

The proofs of Theorem 3 and 4 are given in Appendix $B$ and C. respectively. Since the proof techniques differ significantly, we present the two theorems/proofs separately.

Both theorems are stated for derivatives taken at $t=0$. Nevertheless, the analysis can be extended for arbitrary $t \in[0,1]$, that is, $\frac{d}{d t} \sin ^{2} \theta_{i} \leq 0$ for all $t \in[0,1]$, where the equality holds if and only if $\theta_{i}(t)=0$. To show that this is the case, note that in proving both Theorem 3 and Theorem 4 we constructed a continuous path $\boldsymbol{U}(t)$ such that $\boldsymbol{U}(0)=\boldsymbol{U}_{0}$ and $\boldsymbol{U}(1) \in \mathcal{U}_{G}$. By fixing this continuous path, we observe that:
1) All the $r$ principal angles between $\operatorname{span}\left(\boldsymbol{U}_{0}\right)$ and span $(\boldsymbol{U}(1))$ are monotonically decreasing as $t$ increases to one. This implies that Assumption I holds for all $t \in[0,1]$.

2) We have $\theta_{i}(t)<\pi / 2$ for all $i \in[n]$ and for all $t \in[0, \epsilon)$ for some sufficiently small $\epsilon>0$. This claim can be verified by invoking the facts that $\theta_{i}(0)<\pi / 2$ for all $i \in[n]$ and that $\theta_{i}$ is a continuous functions for all $i \in[n]$. As a result, all $\boldsymbol{U}(t)$ 's, where $t \in[0, \epsilon)$, satisfy Assumptions I and II.

3) For every $t$ in the interval $[0, \epsilon), \boldsymbol{U}(t)$ is the starting point of the geodesic path from $\boldsymbol{U}(t)$ to $\boldsymbol{U}(1)$, which is a part of the geodesic path from $\boldsymbol{U}(0)$ to $\boldsymbol{U}(1)$. Using the same proof techniques as in Appendix $B$ and $C$, it is clear that $\frac{d}{d t} \sin ^{2} \theta_{i}(t) \leq 0$ for all $t \in[0, \epsilon)$. Hence, $\theta_{i}(t) \leq \theta_{i}(0)<\frac{\pi}{2}$ for all $i \in[n]$ and for all $t \in[0, \epsilon)$.

4) The arguments above can be extended. It can be verified that $\theta_{i}(t) \leq \theta_{i}(0)<\pi / 2$ for all $i \in[n]$ and for all $t \in[0,1]$. This implies that $\boldsymbol{U}(t)$ satisfies Assumptions I and II for all $t \in[0,1]$. Hence, $\frac{d}{d t} \sin ^{2} \theta_{i}(t) \leq 0$ for all $i \in[n]$ and all $t \in[0,1]$, where the equality holds if and only if $\theta_{i}(t)=0$. Theorem 2 therefore holds.

A direct consequence of Theorem 2] is that for almost all $\boldsymbol{U}_{0} \in \mathcal{U}_{m, r}$, there exists a continuous path leading to a global minimizer. However, one does not know this path in the process of solving the matrix completion problem. A practical approach is to use a gradient descent method. We consider the following randomized gradient descent algorithm. Let $\boldsymbol{U}^{(i)} \in \mathcal{U}_{m, r}, i=1,2, \cdots$, be the starting point of the $i^{t h}$ iteration. Clearly, $\boldsymbol{U}^{(i)}, i \geq 2$, is also the end point of the $(i-1)^{t h}$ iteration. We generate the sequence of $\boldsymbol{U}^{(i)}$ 's in the following manner.

1) Let $\boldsymbol{U}^{(1)}$ be randomly generated from the isotropic distribution.

2) Set $i=1$. Execute the following iterative process.

a) Compute the gradient $\nabla_{U^{(i)}} f_{G}$.

b) Let $\boldsymbol{U}^{(i)}(t)$ be the geodesic curve starting at $\boldsymbol{U}^{(i)}(0)=\boldsymbol{U}^{(i)}$ with direction $\boldsymbol{H}=-\nabla_{\boldsymbol{U}^{(i)}} f_{G}$.

c) Let $t^{(i) *}$ be such that $\frac{d}{d t} f_{G}\left(t^{(i) *}\right)=0$ and $\frac{d}{d t} f_{G}(t)<0$ for all $t<t^{(i) *}$.

d) Randomly generate a $t^{(i)}$ from the uniform distribution on $\left(0, t^{(i) *}\right)$.

e) Let $\boldsymbol{U}^{(i+1)}=\boldsymbol{U}^{(i)}\left(t^{(i)}\right)$. Let $i=i+1$. Go to Step (a).

Due to the randomness of $\boldsymbol{U}^{(i)}$, all $\boldsymbol{U}^{(i)}$ 's satisfy Assumptions I and II with probability one. The objective function decreases after each iteration. This gradient descent procedure converges to a global minimum as the number of iterations approachs infinity.

Remark 2: Denote the obtained global minimum by $\hat{\boldsymbol{U}}$. It may happen that $\hat{\boldsymbol{U}} \in \mathcal{U}_{G} \backslash \mathcal{U}_{F}$. In this case, the solution is inconsistent with respect to to the standard Frobenius norm. One can use perturbation techniques to move $\hat{U}$ from the boundary of $\mathcal{U}_{F}$ to the interior region of $\mathcal{U}_{F}$. 


\section{B. The General Framework}

For the cases that are not described in Theorem 2, we have the following corollary.

Corollary 1: (General Cases) Let $\boldsymbol{X} \in \mathbb{R}^{m \times n}$ be a rank$r$ matrix. Let $\boldsymbol{U}_{X} \in \mathcal{U}_{G}$ be a global minimum. For each $i \in[n]$, the following statements are true. Let $\boldsymbol{u}_{X, i} \in$ $\operatorname{span}\left(\boldsymbol{U}_{X}\right) \bigcap \operatorname{span}\left(\boldsymbol{B}_{i}\right)$ be a unit norm vector. Let $\boldsymbol{U}_{0} \in \mathcal{U}_{m, r}$ and $\boldsymbol{w}_{i} \in \mathcal{U}_{r, 1}$ be randomly drawn from the corresponding isotropic distributions respectively. Then with probability one, the vector $\boldsymbol{u}_{0, i} \triangleq \boldsymbol{U}_{0} \boldsymbol{w}_{i}$ is not orthogonal to $\boldsymbol{u}_{X, i}$. Suppose that this is true. Define $\theta_{i}=\cos ^{-1}\left\|\mathcal{P}\left(\boldsymbol{u}_{i}(t), \boldsymbol{B}_{i}\right)\right\|_{2}$. There exists a continuous path $\boldsymbol{u}_{i}(t) \in \mathcal{U}_{m, 1}$ such that $\boldsymbol{u}_{i}(0)=\boldsymbol{u}_{0, i}$, $\boldsymbol{u}_{i}(1) \in \operatorname{span}\left(\boldsymbol{U}_{X, i}\right) \bigcap \mathcal{U}_{m, 1}$, and $\frac{d}{d t} \sin ^{2} \theta_{i} \leq 0$, where the equality holds if and only if $\theta_{i}(t)=0$.

Proof: Without loss of generality, we assume that $\left\langle\boldsymbol{u}_{0, i}, \boldsymbol{u}_{X, i}\right\rangle>0$. The desired continuous path is given by

$$
\boldsymbol{u}_{i}(t)=\frac{(1-t) \boldsymbol{u}_{0, i}+t \boldsymbol{u}_{X, i}}{\left\|(1-t) \boldsymbol{u}_{0, i}+t \boldsymbol{u}_{X, i}\right\|}, t \in[0,1] .
$$

The detailed arguments are the same as those in the proof of Theorem 3 , and therefore omitted.

Remark 3: This corollary is similar to Theorems 3 and 4 in the sense that there exist continuous paths along which the atomic functions decreases.

At the same time, Corollary 1 differs from Theorems 3 and 4 in two aspects. First, the paths $\boldsymbol{u}_{i}(t)$ in Corollary 1 may be different for different $i$ 's, while in Theorems 3 and 4, a single continuous path $\boldsymbol{U}(t)$ is constructed. Second, the angle $\theta_{i}$ in Corollay 1 is essentially the principal angle between the 1-dimensional subspace $\operatorname{span}\left(\boldsymbol{u}_{i}(t)\right)$ and the subspace $\operatorname{span}\left(\boldsymbol{B}_{i}\right)$. In contrast, Theorem 3 and 4 involve the minimum principal angle between the $r$-dimensional subspace $\operatorname{span}(\boldsymbol{U}(t))$ and the subspace $\operatorname{span}\left(\boldsymbol{B}_{i}\right)$.

\section{CONCLUSION}

We considered the problem of how to search for a consistent completion of low-rank matrices. We showed that Frobenius norm combined with a projection operator results in a discontinuous objective function and therefore makes gradient descent approach fail. We proposed to replace the Frobenius norm with the chordal distance. The chordal distance is the "best" smooth version of the Frobenius norm in the sense that the solution set of the former is the closure of the solution set of the latter. Based on the chordal distance, we derived strong performance guarantees for two completion scenarios. The derived performance guarantees do not rely on incoherence conditions or large matrix sizes, and they hold with probability one.

\section{APPENDIX}

\section{A. Proof of Theorem 1}

We omit the subscript $i$ to simplify notation. The proof consists of two parts, showing that:

1) $\mathcal{U}_{F} \subset \mathcal{U}_{G}$

2) for any given $\boldsymbol{U}_{0} \in \mathcal{U}_{G}$, there exists a sequence $\left\{\boldsymbol{U}^{(n)}\right\} \subset \mathcal{U}_{F}$ such that $\lim _{n \rightarrow \infty}\left\|\boldsymbol{U}_{0}-\boldsymbol{U}^{(n)}\right\|_{F}=0$.
We start by proving that $\mathcal{U}_{F} \subset \mathcal{U}_{G}$. For any given $U \in \mathcal{U}_{F}$, there exists a nonzero vector $\boldsymbol{w} \in \mathbb{R}^{r}$ such that $\boldsymbol{U}_{\Omega} \boldsymbol{w}=\boldsymbol{x}_{\Omega}$. Let $\boldsymbol{b}=\boldsymbol{U} \boldsymbol{w} /\|\boldsymbol{w}\|$. Clearly, $\|\boldsymbol{b}\|_{F}=1$. Recall the formula for $\boldsymbol{B}_{\boldsymbol{x}_{\Omega}}$. We can write $\boldsymbol{b}$ as a linear combination of columns of $\boldsymbol{B}_{\boldsymbol{x}_{\Omega}}$

$$
\boldsymbol{b}=\frac{1}{\|\boldsymbol{w}\|} \boldsymbol{x}_{\Omega}+\sum_{j \in \Omega^{c}} b_{j} \boldsymbol{e}_{j}=\frac{\left\|\boldsymbol{x}_{\Omega}\right\|}{\|\boldsymbol{w}\|} \overline{\boldsymbol{x}}_{\Omega}+\sum_{j \in \Omega^{c}} b_{j} \boldsymbol{e}_{j} .
$$

As a result,

$$
\left\|\boldsymbol{B}_{\boldsymbol{x}_{\Omega}}^{T} \boldsymbol{b}\right\|_{F}=\left\|\boldsymbol{B}_{\boldsymbol{x}_{\Omega}}^{T} \boldsymbol{U} \frac{\boldsymbol{w}}{\|\boldsymbol{w}\|_{F}}\right\|_{F}=1 .
$$

It follows that the largest singular value of $\boldsymbol{B}_{\boldsymbol{x}_{\Omega}}^{T} \boldsymbol{U}$ is one. Therefore, $\boldsymbol{U} \in \mathcal{U}_{G}$, and we thus have $\mathcal{U}_{F} \subset \mathcal{U}_{G}$.

To prove the second part, we make use of the following notation. For any given $\boldsymbol{U}_{0} \in \mathcal{U}_{G}$, let $\boldsymbol{u}_{1}, \cdots, \boldsymbol{u}_{r}$ be the left singular vectors of the matrix $\boldsymbol{U}_{0} \boldsymbol{U}_{0}^{T} \boldsymbol{B}_{\boldsymbol{x}_{\Omega}}$ corresponding to the $i^{t h}$ largest singular value. Let $k$ be the multiplicity of the singular value one, i.e., the number of singular values that equal to one. Let $\boldsymbol{U}_{1: k}=\left[\boldsymbol{u}_{1}, \cdots, \boldsymbol{u}_{k}\right]$ and $\boldsymbol{U}_{k+1: r}=\left[\boldsymbol{u}_{k+1}, \cdots, \boldsymbol{u}_{r}\right]$. Clearly, $\lambda_{\max }\left(\boldsymbol{U}_{k+1: r}^{T} \boldsymbol{B}_{\boldsymbol{x}_{\Omega}}\right)<1$.

It suffices to focus on $\boldsymbol{U}$ instead of $\boldsymbol{U}_{0}$. That is, to prove the second part, it suffices to find a sequence in $\mathcal{U}_{F}$ converging to $\boldsymbol{U}$. To verify this claim, let $\boldsymbol{V}=\boldsymbol{U}^{T} \boldsymbol{U}_{0}$. Then $\boldsymbol{V} \in \mathcal{U}_{r, r}$ and $\boldsymbol{U}_{0}=\boldsymbol{U} \boldsymbol{V}$. Suppose that $\left\{\boldsymbol{U}^{(n)}\right\} \subset \mathcal{U}_{F}$ is a sequence such that $\boldsymbol{U}^{(n)} \rightarrow \boldsymbol{U}$. It is clear that $\boldsymbol{U}^{(n)} \boldsymbol{V} \rightarrow \boldsymbol{U} \boldsymbol{V}=\boldsymbol{U}_{0}$. Furthermore, since

$\boldsymbol{x}_{\Omega}=\boldsymbol{U}_{\Omega}^{(n)} \boldsymbol{w}^{(n)}=\boldsymbol{U}_{\Omega}^{(n)} \boldsymbol{V}\left(\boldsymbol{V}^{T} \boldsymbol{w}^{(n)}\right)=\left(\boldsymbol{U}^{(n)} \boldsymbol{V}\right)_{\Omega} \boldsymbol{w}^{\prime(n)}$,

one has $\boldsymbol{U}^{(n)} \boldsymbol{V} \in \mathcal{U}_{F}$. The sequence $\left\{\boldsymbol{U}^{(n)} \boldsymbol{V}\right\} \subset \mathcal{U}_{F}$ is the desired sequence that converges to $\boldsymbol{U}_{0}$. It is also important to note that $\boldsymbol{U} \in \mathcal{U}_{G}$, since

$$
\lambda\left(\boldsymbol{U}_{0} \boldsymbol{U}_{0}^{T} \boldsymbol{B}_{\boldsymbol{x}_{\Omega}}\right)=\lambda\left(\boldsymbol{U} \boldsymbol{V} \boldsymbol{V}^{T} \boldsymbol{U}^{T} \boldsymbol{B}_{\boldsymbol{x}_{\Omega}}\right)=\lambda\left(\boldsymbol{U} \boldsymbol{U}^{T} \boldsymbol{B}_{\boldsymbol{x}_{\Omega}}\right) .
$$

We claim that

$$
\boldsymbol{U} \in \mathcal{U}_{F} \text { if and only if } \boldsymbol{U}_{1: k, \Omega} \neq \mathbf{0} \text {. }
$$

To prove this claim, we shall show that

$$
\boldsymbol{U}_{1: k, \Omega} \neq \mathbf{0} \Rightarrow \boldsymbol{U} \in \mathcal{U}_{F}
$$

and

$$
\boldsymbol{U}_{1: k, \Omega}=\mathbf{0} \Rightarrow \boldsymbol{U} \notin \mathcal{U}_{F} .
$$

To prove (15), suppose that $\boldsymbol{U}_{1: k, \Omega} \neq 0$. Without loss of generality, let $\boldsymbol{u}_{1, \Omega} \neq \mathbf{0}$. Since $\boldsymbol{u}_{1}$ is the left singular vector corresponding to the singular value equal to one, $\boldsymbol{u}_{1}$ can be written as a linear combination of the columns of $\boldsymbol{B}_{\boldsymbol{x}_{\Omega}}: \boldsymbol{u}_{1}=$ $a_{1} \overline{\boldsymbol{x}}_{\Omega}+\sum_{j \in \Omega^{c}} a_{j} \boldsymbol{e}_{j}$. Since $\boldsymbol{u}_{1, \Omega}=a_{1} \overline{\boldsymbol{x}}_{\Omega} \neq \mathbf{0}$, one has $a_{1} \neq$ 0 . As a result, $\boldsymbol{x}_{\Omega}=a \boldsymbol{u}_{1, \Omega}$ for some constant $a \neq 0$. Hence, $d_{F}\left(\boldsymbol{x}_{\Omega}, \boldsymbol{U}\right)=0$ and $\boldsymbol{U} \in \mathcal{U}_{F}$.

To prove (16), assume that $\boldsymbol{U}_{1: k, \Omega}=\mathbf{0}$. Since $\mathcal{P}\left(\boldsymbol{x}_{\Omega}, \boldsymbol{U}_{\Omega}\right)=\mathcal{P}\left(\boldsymbol{x}_{\Omega}, \boldsymbol{U}_{k+1: r, \Omega}\right)$, proving that $\boldsymbol{U} \notin \mathcal{U}_{F}$ is equivalent to proving that $\boldsymbol{x}_{\Omega}-\mathcal{P}\left(\boldsymbol{x}_{\Omega}, \boldsymbol{U}_{k+1: r, \Omega}\right) \neq \mathbf{0}$. This inequality can be proved by contradiction. Suppose that we have an equality. Then there exists a vector $\boldsymbol{w} \in \mathbb{R}^{r-k}$ such that $\boldsymbol{U}_{k+1: r, \Omega} \boldsymbol{w}=\boldsymbol{x}_{\Omega}$. Let $\boldsymbol{b}=\boldsymbol{U}_{k+1: r} \boldsymbol{w} /\|\boldsymbol{w}\|$. It is straightforward to show (using similar arguments as the ones 
used for proving $\left.\mathcal{U}_{F} \subset \mathcal{U}_{G}\right)$ that $\boldsymbol{b} \in \operatorname{span}\left(\boldsymbol{B}_{\boldsymbol{x}_{\Omega}}\right)$ and the largest singular value of $\boldsymbol{U}_{k+1: r}^{T} \boldsymbol{B}_{\boldsymbol{x}_{\Omega}}$ is one. This contradicts the fact that $\lambda_{\max }\left(\boldsymbol{U}_{k+1: r}^{T} \boldsymbol{B}_{\boldsymbol{x}_{\Omega}}\right)<1$.

Now we are ready to construct a sequence in $\mathcal{U}_{F}$ converging to $\boldsymbol{U}$. If $\boldsymbol{U}_{1: k, \Omega} \neq 0$, then $\boldsymbol{U} \in \mathcal{U}_{F}$ and it is trivial to find a sequence in $\mathcal{U}_{F}$ converging to $U$. It remains to find a sequence $\left\{\boldsymbol{U}^{(n)}\right\} \subset \mathcal{U}_{F}$ that converges to $\boldsymbol{U}$ when $\boldsymbol{U}_{1: k, \Omega}=0$. Define $\boldsymbol{x}_{r}=\boldsymbol{x}_{\Omega}-\mathcal{P}\left(\boldsymbol{x}_{\Omega}, \boldsymbol{U}_{\Omega}\right)$. Since $\boldsymbol{U}_{1: k, \Omega}=0$, one has $\boldsymbol{U} \notin \mathcal{U}_{F}$ and $\boldsymbol{x}_{r} \neq \mathbf{0}$. Note that $\boldsymbol{x}_{r, \Omega^{c}}=\mathbf{0}$ and that $\boldsymbol{x}_{r, \Omega} \perp \boldsymbol{u}_{i, \Omega}$ for all $i \in[r]$. It can be verified that $\boldsymbol{x}_{r} \perp \boldsymbol{u}_{1}, \cdots, \boldsymbol{x}_{r} \perp \boldsymbol{u}_{r}$. Let

$$
\boldsymbol{U}_{\epsilon}=\left[\frac{\boldsymbol{u}_{1}+\epsilon \boldsymbol{x}_{r}}{\sqrt{1+\epsilon^{2}}}, \boldsymbol{u}_{2}, \cdots, \boldsymbol{u}_{r}\right] .
$$

It can be verified that $\boldsymbol{U}_{\epsilon} \in \mathcal{U}_{m, r}$. Furthermore, $\mathcal{P}\left(\boldsymbol{x}_{\Omega}, \boldsymbol{U}_{\Omega}\right)=$ $\mathcal{P}\left(\boldsymbol{x}_{\Omega},\left[\boldsymbol{x}_{r}, \boldsymbol{U}_{k+1: r, \Omega}\right]\right)=\boldsymbol{x}_{\Omega}$ and therefore $\boldsymbol{U}_{\epsilon} \in \mathcal{U}_{F}$ for all $\epsilon \neq 0$. Now choose a sequence $\left\{\boldsymbol{U}^{(n)}\right\}=\left\{\boldsymbol{U}_{1 / n}\right\}$. It is a sequence in $\mathcal{U}_{F}$ and it converges to $\boldsymbol{U}$. This completes the proof.

\section{B. Proof of Theorem 3}

Since $\boldsymbol{X}_{\Omega}$ is generated from a rank-one matrix, there exists a $\boldsymbol{u}_{\boldsymbol{X}} \in \mathcal{U}_{m, 1}$ such that $\boldsymbol{u}_{\boldsymbol{X}} \in \operatorname{span}\left(\boldsymbol{B}_{i}\right)$ for all $i \in[n]$. Without loss of generality, we assume $\left\langle\boldsymbol{u}, \boldsymbol{u}_{\boldsymbol{X}}\right\rangle>0$ : by Assumption I, $\left\langle\boldsymbol{u}, \boldsymbol{u}_{\boldsymbol{X}}\right\rangle \neq 0$; if $\left\langle\boldsymbol{u}, \boldsymbol{u}_{\boldsymbol{X}}\right\rangle<0$, we replace $\boldsymbol{u}_{\boldsymbol{X}}$ with $-\boldsymbol{u}_{\boldsymbol{X}}$.

Now define

$$
\boldsymbol{u}(t)=\frac{(1-t) \boldsymbol{u}_{0}+t \boldsymbol{u}_{\boldsymbol{X}}}{\left\|(1-t) \boldsymbol{u}_{0}+t \boldsymbol{u}_{\boldsymbol{X}}\right\|}=\frac{(1-t) \boldsymbol{u}_{0}+t \boldsymbol{u}_{\boldsymbol{X}}}{L(t)},
$$

where $L(t) \triangleq\left\|(1-t) \boldsymbol{u}_{0}+t \boldsymbol{u}_{\boldsymbol{X}}\right\|$. Clearly $\boldsymbol{u}(0)=\boldsymbol{u}_{0}$ and $\boldsymbol{u}(t) \in \mathcal{U}_{m, 1}$ in a neighborhood of $t=0$.

For every $i \in[n]$, we shall show that

$$
\left.\frac{d}{d t}\right|_{t=0} \sin ^{2} \theta_{i}=-\left.2 \frac{d}{d t}\right|_{t=0}\left(\frac{1}{2} \cos ^{2} \theta_{i}\right) \leq 0,
$$

where the equality holds if and only if $\theta_{i}=0$. Let $\mathcal{P}_{i} \boldsymbol{u}$ denote the vector $\mathcal{P}\left(\boldsymbol{u}, \boldsymbol{B}_{i}\right)=\boldsymbol{B}_{i} \boldsymbol{B}_{i}^{T} \boldsymbol{u}$. Since $\boldsymbol{u}_{\boldsymbol{X}} \in \operatorname{span}\left(\boldsymbol{B}_{i}\right)$, one has

$$
\mathcal{P}_{i} \boldsymbol{u}=\frac{1}{L(t)}\left((1-t) \mathcal{P}_{i} \boldsymbol{u}_{0}+t \boldsymbol{u}_{\boldsymbol{X}}\right) .
$$

We then have

$$
\begin{aligned}
\left.\frac{d}{d t}\right|_{t=0}\left(\frac{1}{2} \cos ^{2} \theta_{i}\right)=\left.\frac{d}{d t}\right|_{t=0} \frac{1}{2}\left\|\mathcal{P}_{i} \boldsymbol{u}\right\|^{2} \\
=\left.\frac{d}{d t}\right|_{t=0}\left[\frac{1}{2}\left(\frac{1-t}{L(t)}\right)^{2}\left\|\mathcal{P}_{i} \boldsymbol{u}_{0}\right\|^{2}\right. \\
\left.\quad+\frac{1}{2}\left(\frac{t}{L(t)}\right)^{2}+\frac{\left(t-t^{2}\right)}{L^{2}(t)}\left\langle\mathcal{P}_{i} \boldsymbol{u}_{0}, \boldsymbol{u}_{\boldsymbol{X}}\right\rangle\right] \\
=\left(-1-L^{\prime}(0)\right)\left\|\mathcal{P}_{i} \boldsymbol{u}_{0}\right\|^{2}+\left\langle\mathcal{P}_{i} \boldsymbol{u}_{0}, \boldsymbol{u}_{\boldsymbol{X}}\right\rangle .
\end{aligned}
$$

Note that

$$
\left\langle\mathcal{P}_{i} \boldsymbol{u}_{0}, \boldsymbol{u}_{\boldsymbol{X}}\right\rangle=\boldsymbol{u}_{\boldsymbol{X}}^{T} \boldsymbol{B}_{i} \boldsymbol{B}_{i}^{T} \boldsymbol{u}_{0}=\left\langle\boldsymbol{u}_{0}, \mathcal{P}_{i} \boldsymbol{u}_{\boldsymbol{X}}\right\rangle=\left\langle\boldsymbol{u}_{0}, \boldsymbol{u}_{\boldsymbol{X}}\right\rangle .
$$

Consequently,

$$
\left.\frac{d}{d t}\right|_{t=0}\left(\frac{1}{2} \cos ^{2} \theta_{i}\right)=\left(-1-L^{\prime}(0)\right)\left\|\mathcal{P}_{i} \boldsymbol{u}_{0}\right\|^{2}+\left\langle\boldsymbol{u}_{0}, \boldsymbol{u}_{\boldsymbol{X}}\right\rangle .
$$

The term $L^{\prime}(0)$ can be computed as follows. Note that

$$
\begin{aligned}
L^{2}(t) & =(1-t)^{2}\left\|\boldsymbol{u}_{0}\right\|^{2}+t^{2}\left\|\boldsymbol{u}_{\boldsymbol{X}}\right\|+2\left(t-t^{2}\right)\left\langle\boldsymbol{u}_{0}, \boldsymbol{u}_{\boldsymbol{X}}\right\rangle \\
& =1-2 t+2 t^{2}+2\left(t-t^{2}\right)\left\langle\boldsymbol{u}_{0}, \boldsymbol{u}_{\boldsymbol{X}}\right\rangle .
\end{aligned}
$$

Therefore,

$$
\left.\frac{d}{d t}\right|_{t=0} L^{2}(t)=-2+2\left\langle\boldsymbol{u}_{0}, \boldsymbol{u}_{\boldsymbol{X}}\right\rangle=2 L(0) L^{\prime}(0) .
$$

As a result,

$$
L^{\prime}(0)=-1+\left\langle\boldsymbol{u}_{0}, \boldsymbol{u}_{\boldsymbol{X}}\right\rangle .
$$

Substituting (19) into (18) one can see that

$$
\left.\frac{d}{d t}\right|_{t=0}\left(\frac{1}{2} \cos ^{2} \theta_{i}\right)=\left\langle\boldsymbol{u}_{0}, \boldsymbol{u}_{\boldsymbol{X}}\right\rangle\left(1-\left\|\mathcal{P}_{i} \boldsymbol{u}_{0}\right\|^{2}\right) \geq 0,
$$

where the equality holds if and only if $\left\|\mathcal{P}_{i} \boldsymbol{u}_{0}\right\|=1$, i.e., $\boldsymbol{u}_{0} \in \operatorname{span}\left(\boldsymbol{B}_{i}\right)$ and $\theta_{i}=0$. This completes the proof.

\section{Proof of Theorem 4}

Let $\boldsymbol{U}_{\boldsymbol{X}} \in \mathcal{U}_{m, r}$ be such that every column of $\boldsymbol{X}$ is in the subspace $\operatorname{span}\left(\boldsymbol{U}_{\boldsymbol{X}}\right)$. Consider the compact singular decomposition $\boldsymbol{U}_{0} \boldsymbol{U}_{0}^{T} \boldsymbol{U}_{\boldsymbol{X}} \boldsymbol{U}_{\boldsymbol{X}}^{T}=\boldsymbol{U}_{0}^{\prime} \boldsymbol{S} \boldsymbol{U}_{\boldsymbol{X}}^{\prime T}$, where $\boldsymbol{S} \in \mathbb{R}^{r \times r}$ is the diagonal matrix containing the singular values and $\boldsymbol{U}_{0}^{\prime}$ and $\boldsymbol{U}_{\boldsymbol{X}}^{\prime}$ are the left and right singular vector matrices, respectively. Clearly, $\boldsymbol{U}_{0}$ and $\boldsymbol{U}_{0}^{\prime}$ generate the same subspace, and so do $\boldsymbol{U}_{\boldsymbol{X}}$ and $\boldsymbol{U}_{\boldsymbol{X}}^{\prime}$. For simplicity, we present our proof for $\boldsymbol{U}_{0}^{\prime}$ and $\boldsymbol{U}_{\boldsymbol{X}}^{\prime}$ and omit the superscripts. With this simplification, one has $\boldsymbol{U}^{T} \boldsymbol{U}_{\boldsymbol{X}}=\boldsymbol{S}=\operatorname{diag}\left(\left[\lambda_{1}, \cdots, \lambda_{r}\right]\right)$.

For the $i^{\text {th }}$ column of $\boldsymbol{X}$, we compute $\nabla_{\boldsymbol{U}_{0}} \cos \theta_{i}$. Since we are considering the full sampling case, we have $\boldsymbol{B}_{i}=\overline{\boldsymbol{x}}_{i}$. Because $\overline{\boldsymbol{x}}_{i} \in \operatorname{span}\left(\boldsymbol{U}_{\boldsymbol{X}}\right)$, there exists $\overline{\boldsymbol{w}} \in \mathcal{U}_{r, 1}$ such that $\overline{\boldsymbol{x}}_{i}=\boldsymbol{U}_{\boldsymbol{X}} \overline{\boldsymbol{w}}$. To compute $\nabla_{\boldsymbol{U}_{0}} \cos \theta_{i}$, we need the first left and the first right singular vectors of the matrix $\overline{\boldsymbol{x}}_{i} \overline{\boldsymbol{x}}_{i}^{T} \boldsymbol{U}_{0}$. The first left singular vector is clearly $\overline{\boldsymbol{x}}_{i}$ and the first right singular vector equals $\boldsymbol{U}_{0}^{T} \overline{\boldsymbol{x}}_{i}=\boldsymbol{U}_{0}^{T} \boldsymbol{U}_{\boldsymbol{X}} \overline{\boldsymbol{w}}=\boldsymbol{S} \overline{\boldsymbol{w}}$. Hence,

$$
\begin{aligned}
\nabla_{\boldsymbol{U}_{0}} \cos \theta_{i} & =\left(\boldsymbol{I}-\boldsymbol{U}_{0} \boldsymbol{U}_{0}^{T}\right) \overline{\boldsymbol{x}}_{i} \overline{\boldsymbol{w}}^{T} \boldsymbol{S}^{T} \\
& =\left(\boldsymbol{I}-\boldsymbol{U}_{0} \boldsymbol{U}_{0}^{T}\right) \boldsymbol{U}_{\boldsymbol{X}} \overline{\boldsymbol{w}} \overline{\boldsymbol{w}}^{T} \boldsymbol{S}^{T} .
\end{aligned}
$$

According to Lemma 1, $\left(\boldsymbol{I}-\boldsymbol{U}_{0} \boldsymbol{U}_{0}^{T}\right) \boldsymbol{U}_{\boldsymbol{X}}$ can be written as $\boldsymbol{G} \operatorname{diag}\left(\left[\sin \alpha_{1}, \cdots, \sin \alpha_{j}\right]\right)$, where $\boldsymbol{G}=\left[\boldsymbol{g}_{1}, \cdots, \boldsymbol{g}_{r}\right] \in$ $\mathcal{U}_{m, r}$, and $\alpha_{i}=\cos ^{-1} \lambda_{i}$ 's, $i=1, \cdots, r$, are the principal angles between $\operatorname{span}\left(\boldsymbol{U}_{0}\right)$ and $\operatorname{span}\left(\boldsymbol{U}_{\boldsymbol{X}}\right)$.

We consider the geodesic $\boldsymbol{U}(t)$ from $\boldsymbol{U}_{0}$ to $\boldsymbol{U}_{\boldsymbol{X}}$. In Lemma 1 (part 1), we show that this geodesic is given by the $\boldsymbol{U}(t)$ satisfying $\boldsymbol{U}(0)=\boldsymbol{U}_{0}$ and $\dot{\boldsymbol{U}}(0)=\boldsymbol{G} \operatorname{diag}\left(\left[\alpha_{1}, \cdots, \alpha_{r}\right]\right)$. Along this path, we have

$$
\begin{aligned}
& \left.\frac{d}{d t}\right|_{t=0} \cos \theta_{i}=\left\langle\nabla_{\boldsymbol{U}_{0}} \cos \theta_{i}, \boldsymbol{G} \operatorname{diag}\left(\left[\alpha_{1}, \cdots, \alpha_{r}\right]\right)\right\rangle \\
= & \operatorname{trace}\left(\left(\boldsymbol{G} \operatorname{diag}\left(\left[\alpha_{1}, \cdots, \alpha_{r}\right]\right)\right)^{T}\right. \\
& \left.\left(\left(\boldsymbol{I}-\boldsymbol{U}_{0} \boldsymbol{U}_{0}^{T}\right) \boldsymbol{U}_{\boldsymbol{X}}\right) \overline{\boldsymbol{w}} \overline{\boldsymbol{w}}^{T} \boldsymbol{S}^{T}\right) \\
= & \operatorname{trace}\left(\operatorname{diag}\left(\left[\cdots, \alpha_{j} \sin \alpha_{j}, \cdots\right]\right) \overline{\boldsymbol{w}} \overline{\boldsymbol{w}}^{T} \boldsymbol{S}\right) \\
= & \operatorname{trace}\left(\left(\boldsymbol{I}-\boldsymbol{S}^{2}\right) \overline{\boldsymbol{w}} \overline{\boldsymbol{w}}^{T} \boldsymbol{S}\right) \\
= & \sum_{j=1}^{r} \bar{w}_{j}^{2} \alpha_{j} \sin \alpha_{j} \cos \alpha_{j} \geq 0 .
\end{aligned}
$$


We claim that under Assumption II, equality in (20) holds if and only if $\theta_{i}=0$. If $\theta_{i}=0$, then $\overline{\boldsymbol{x}}_{i} \in \operatorname{span}\left(\boldsymbol{U}_{0}\right)$. According to Lemma 1 (part 2), $\bar{w}_{j}=0$ for all $j$ such that $\alpha_{j} \neq 0$. The equality in (20) thus holds. Otherwise, if $\theta_{i} \neq 0$, then $\overline{\boldsymbol{x}}_{i} \notin \operatorname{span}\left(\boldsymbol{U}_{0}\right)$. Again, according to Lemma 1 (part 2), there exists an $j \in[r]$ such that $\alpha_{i}>0$ and $\bar{w}_{j} \neq 0$. Hence, we have a strict inequality in 20. Finally, note that

$$
\left.\frac{d}{d t}\right|_{t=0} \sin ^{2} \theta_{i}=-\left.2 \frac{d}{d t}\right|_{t=0} \cos \theta_{i} \leq 0 .
$$

This proves the theorem.

\section{REFERENCES}

[1] D. Donoho, "Compressed sensing," IEEE Trans. Inform. Theory, vol. 52, no. 4, pp. 1289-1306, 2006.

[2] E. Candès, J. Romberg, and T. Tao, "Robust uncertainty principles: exact signal reconstruction from highly incomplete frequency information," IEEE Trans. Inform. Theory, vol. 52, no. 2, pp. 489-509, 2006.

[3] E. Candès and T. Tao, "Decoding by linear programming," Information Theory, IEEE Transactions on, vol. 51, no. 12, pp. 4203-4215, 2005.

[4] B. Recht, M. Fazel, and P. A. Parrilo, "Guaranteed minimum-rank solutions of linear matrix equations via nuclear norm minimization," arXiv:0706.4138, 2007.

[5] E. Candes and B. Recht, "Exact matrix completion via convex optimization," arXiv:0805.4471, 2008.

[6] E. J. Candes and T. Tao, "The power of convex relaxation: Near-optimal matrix completion," arXiv:0903.1476, Mar. 2009.

[7] V. Chandrasekaran, S. Sanghavi, P. A. Parrilo, and A. S. Willsky, "Ranksparsity incoherence for matrix decomposition," arXiv:0906.2220.

[8] E. J. Candes and Y. Plan, "Matrix completion with noise," arXiv:0903.3131, Mar. 2009.

[9] J. Cai, E. J. Candes, and Z. Shen, "A singular value thresholding algorithm for matrix completion," arXiv:0810.3286, 2008.

[10] K. Lee and Y. Bresler, "ADMiRA: atomic decomposition for minimum rank approximation," arXiv:0905.0044, Apr. 2009.

[11] W. Dai and O. Milenkovic, "Subspace pursuit for compressive sensing signal reconstruction," IEEE Trans. Inform. Theory, vol. 55, pp. 2230 2249, May 2009.

[12] D. Needell and J. A. Tropp, "CoSaMP: Iterative signal recovery from incomplete and inaccurate samples," Applied and Computational Harmonic Analysis, vol. 26, pp. 301-321, May 2009.

[13] R. Meka, P. Jain, and I. S. Dhillon, "Guaranteed rank minimization via singular value projection," arXiv:0909.5457, 2009.

[14] T. Blumensath and M. E. Davies, "Iterative hard thresholding for compressed sensing," Applied and Computational Harmonic Analysis, vol. 27, pp. 265-274, Nov. 2009.

[15] J. Haldar and D. Hernando, "Rank-constrained solutions to linear matrix equations using powerfactorization," IEEE Signal Processing Letters, pp. 16:584-587, 2009.

[16] R. H. Keshavan, A. Montanari, and S. Oh, "Matrix completion from a few entries," arXiv:0901.3150, 2009.

[17] W. Dai and O. Milenkovic, "SET: an algorithm for consistent matrix completion," in IEEE International Conf. on Acoustics, Speech, and Signal Processing (ICASSP), March 2010.

[18] A. Edelman, T. Arias, and S. T. Smith, "The geometry of algorithms with orthogonality constraints," SIAM Journal on Matrix Analysis and Applications, vol. 20, pp. 303-353, April 1999.

[19] J. H. Conway, R. H. Hardin, and N. J. A. Sloane, "Packing lines, planes, etc., packing in Grassmannian spaces," Exper. Math., vol. 5, pp. 139159, 1996.

[20] W. Dai, Y. Liu, and B. Rider, "Quantization bounds on grassmann manifolds and applications to mimo communications," IEEE Trans. on Inform. Theory, vol. 54, pp. $1108-1123$, march 2008.

[21] A. T. James, "Normal multivariate analysis and the orthogonal group," Ann. Math. Statist., vol. 25, no. 1, pp. 40 - 75, 1954.

[22] M. Adler and P. van Moerbeke, "Integrals over Grassmannians and random permutations," Advances in Mathematics, vol. 181, no. 1, pp. 190-249, 2004.

[23] W. Dai, B. Rider, and Y. Liu, "Volume growth and general rate quantization on grassmann manifolds," in IEEE Global Telecommunications Conference (Globecom), pp. 1441 -1445, Nov. 26-30 2007. 